\title{
Time to experiment...
}

Gráinne Conole, Martin Oliver and Jane Seale

email:g.conole@bristol.ac.uk

In the last edition of $A L T-J$, the editorial team decided to try an experiment by creating a more interactive and critical debate on a particular article with the journal. Readers were invited to submit comments on an article by Davis and Denning entitled: 'Almost as helpful as good theory: some conceptual possibilities for the online classroom' (Davis and Denning, 2000). Summarized below are some of the key comments raised about the paper. Many thanks to everyone who took the time to send in these comments; individual acknowledgements have not been included for reasons of confidentiality.

\section{Readers' comments}

Comments fell into four main categories. The first addressed the paper's contribution to the learning technology field. Some readers felt the paper to be quite difficult to understand, perhaps because it seems preliminary and speculative. One reader commented that in many respects Davis and Denning were exploring surprisingly uncharted waters. Others added the following comments and observations:

The paper is interesting in that it provides a framework for thinking about 'dimensions of practice' in virtual learning environments. This is timely and the area certainly lacks robust theoretical frameworks of the sort that this paper is trying to devise.

This is an insightful categorization of typologies of 'group function in cyberspace'. The six conceptual areas, and the discussion of group function (and dysfunction) in each area will provide a useful framework for future researchers.

Some of the requests were simply for additional context: 
Mention is made at several points in the paper of 'action science groups' - this seems to be an important aspect of their work and would benefit from fuller exposition, as many readers will not be familiar with 'action science groups'.

There are several places where claims are made which would benefit from further substantiation either by reference to the literature or their own data.

The distinction between different Basic Assumption groups and Work groups was not clear and needs to be made more explicit.

Others, however, raised questions of interpretation and application:

The paper identifies that the theorizing it contains is based upon 'ongoing action research' over a three-year period. However, there is very little explicit connection made between the framework and the theoretical analysis that is provided with this underlying data. This is reflected by the comment in the conclusion that the model/framework that is presented is 'the product of inspiration and intuition based on our iterative analysis of data'. The relationship to the data should really be drawn out more strongly throughout the paper - perhaps simply by including some illustrative quotes/examples.

The paper describes 'two main theoretical sources' (group dynamics and situated learning). Whilst the group dynamics thread does permeate the paper, the situated learning thread was much less clear. For example, there seems to be a leap from situated learning to learning dynamics in Table 1.

Some more analysis of the interaction between 'conceptual areas and their corresponding states' and 'types' (based on the data rather than on supposition) is needed before the model can be thought to be robust. However, the authors seem to be aware of this and the framework does provide a useful starting point for analysis even prior to this work taking place.

The authors use scaffolding to mean 'students building upon one another's comments'. This is a rather restricted way of using this term, which is more usually used (within the context of learning) to mean providing support for learners (through the means of channels) - stemming from the work of Vygotsky.

The title of the paper suggests a playful reference to Kurt Lewin ('nothing so practical as a good theory') but there is little evidence in the paper of ways of evaluating the utility of the 'conceptual possibilities' alluded to in the title. Indeed, it was rather hard to say exactly what these might be.

Some of the language was a little off-putting - why use ersatz psychology when there are plenty of good and relevant constructs in scientific psychology?

Finally, there were some comments that challenged aspects of the paper, such as the following:

There is concern about whether the $2 \times 2$ matrix is an appropriate extrapolation from the six conceptual areas. It appears that in fact there are already six dimensions to the matrix. From these six dimensions, four typologies are observed which are then reduced (back-mapped) to the two axes. A more common use of the $2 \times 2$ matrix is to characterize the points on the two scales and then build the representation of the quadrants from the 
positions of the respondents along the $x$ and $y$ axes respectively. This is not in any way to suggest that the four typologies are not both accurate and useful. It does, however question how the addition of two more dimensions 'depth' and 'time' will be achieved. One alternative to retaining the $2 \times 2$-matrix model and to testing and extending it would be to characterize each axis formally as 'Learning Dynamics' and 'Group Dynamics' (in cyberspace). Alternatively, researchers might wish to work with the underlying 6dimension array. If a visual representation of outputs was required, spider diagrams could be used instead of $2 \times 2$ matrices. If researchers used consistent scales along the six dimensions, each of the typologies would have a characteristic 'shape'. Other groups could then be analysed and their 'shapes' compared with the four typologies.

These last questions and issues are, perhaps, the most interesting to focus upon - this experiment was intended to promote debate, rather than consensus! In the following article, Davis and Denning respond to these comments. We would be very interested to hear readers' views on the value of this form of more interactive, in-depth discussion on one of the papers. Please email us with your thoughts.

\section{References}

Davis, M. and Denning, K. (2000), 'Almost as helpful as good theory: some conceptual possibilities for the online classroom', $A L T-J, 9$ (2), 64-75. 